\title{
Gradhiva
}

GRADHIV

Revue d'anthropologie et d'histoire des arts

$2 \mid 2005$

Autour de Lucien Sebag

\section{Le discours du mythe}

The Discourse of the myth

Salvatore D'Onofrio

\section{(2) OpenEdition}

Journals

Édition électronique

URL : http://journals.openedition.org/gradhiva/529

DOI : 10.4000/gradhiva.529

ISSN : 1760-849X

\section{Éditeur}

Musée du quai Branly Jacques Chirac

\section{Édition imprimée}

Date de publication : 1 novembre 2005

Pagination : 69-87

ISBN : 2-915-133-10-7

ISSN : 0764-8928

\section{Référence électronique}

Salvatore D'Onofrio, «Le discours du mythe », Gradhiva [En ligne], 2 | 2005, mis en ligne le 10 décembre 2008, consulté le 10 décembre 2020. URL : http://journals.openedition.org/gradhiva/529 ; DOI : https://doi.org/10.4000/gradhiva.529

Ce document a été généré automatiquement le 10 décembre 2020.

(c) musée du quai Branly 


\section{Le discours du mythe}

The Discourse of the myth

\section{Salvatore D'Onofrio}

Un mythe se rapporte toujours à des événements passés « avant la création du monde » ou "pendant les premiers âges », en tout cas « il y a longtemps ». Mais la valeur intrinsèque attribuée au mythe provient de ce que les événements, censés se dérouler à un moment du temps, forment aussi une structure permanente. Celle-ci se rapporte simultanément au passé, au présent et au

futur.

Claude Lévi-Strauss, Anthropologie structurale. Un mythe, par exemple, répond à certains besoins, remplit dans une société donnée des fonctions déterminées ; il rattache le devenir présent de la communauté humaine à une Histoire primordiale que la vie profane ne fera que répéter; il permet à

chaque acte humain, à chaque geste, à chaque parole de s'inscrire dans un ordre symbolique qui leur donne sens ; il double l'ordre laïc d'un ordre sacré qui le fonde et inscrit la société dans une continuité qui outrepasse chacun des moments particuliers de son existence. Lucien Sebag, Marxisme et structuralisme. La méthode structurale suppose que l'ethnologue

[...] se décentre par rapport à sa culture. [...] Il rejoint là une des dimension essentielles de

l'ascèse ethnologique qui conduit à l'éclatement des cadres mentaux qui nous sont les plus familiers

et auxquels nous avons tendance à accorder la 
permanence des univers naturels pour dévoiler la possibilité pour l'homme de structurer sa propre existence suivant des principes antithétiques. Lucien Sebag, L'Invention du monde chez les Indiens pueblos. 
1 Déchiré entre la violence qui relève $d u$ « chaos affectif » et le discours de la raison qui se conforme aux normes du savoir, Lucien Sebag interroge encore de nos jours les anthropologues. Depuis sa disparition, la question se pose en effet de comprendre pourquoi la science pour laquelle il était déjà bien plus qu'une promesse n'a pas fait rempart aux pulsions négatives qui l'entraînèrent, à l'âge de trente et un ans (le 9 janvier 1965), dans le tourbillon de la mort. Pourquoi l'anthropologie n'est-elle

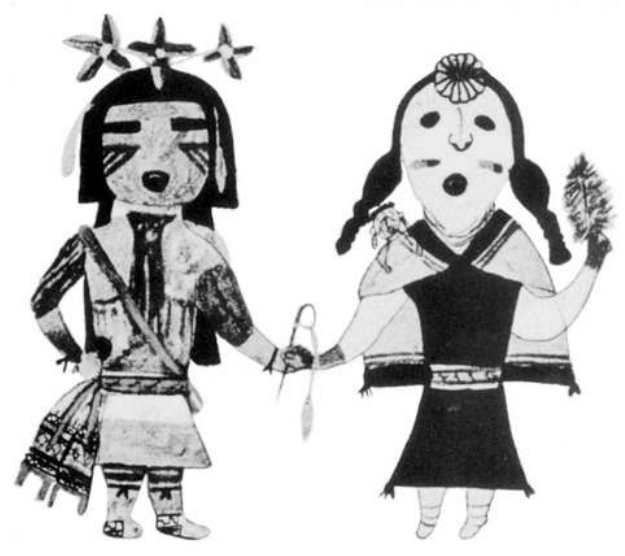
pas parvenue à l'emporter sur la fragilité d'un homme qui avait fini par lui consacrer son existence tout entière ? Le dilemme était toujours en lui, comme en témoignent les premières lignes de l'unique ouvrage publié de son vivant, Marxisme et structuralisme (qu'il dédie à Judith, sa fiancée) : «Le premier pas seul est décisif : du discours ou de la violence, du chaos affectif ou de la raison, que dois-je choisir ?» (1964b) Il avait cependant espéré avoir résolu cette « question initiale » par le pouvoir normatif de l'écriture - « et elle l'est puisque j'écris », affirmait-il.

2 Il y a peut-être là une des clefs non seulement de la dissolution de l'ordre qui le conduisit au suicide, mais encore d'une sorte d'inachèvement de cet «itinéraire spirituel » qui l'avait pourtant enraciné dans l'anthropologie en l'éloignant progressivement du marxisme et, d'une toute autre manière, aussi de la psychanalyse.

Il y a cette illusion de l'écrit, d'une part. Car, s'il est vrai, comme l'affirme Borges, que le livre est une extension de la mémoire et de l'imagination (nous projetant du reste dans l'avenir), il n'en est pas moins vrai qu'il renvoie, justement par l'écriture, à la mort des événements en les fixant à jamais dans le déroulement incessant du temps. D'autre part, la transmutation psychologique couramment assurée par l'expérience ethnographique n'a pas empêché que d'autres questionnements s'imposent. Cela ne veut pas dire qu'une difficulté à dépasser le marxisme et la psychanalyse soit à l'origine du choix ultime de Sebag. Mais, plutôt, que les engagements qu'ils impliquent et qui demeurèrent probablement jusqu'à la fin dans son esprit ont pu affaiblir la charpente qui aurait dû le protéger.

Quant au marxisme, cette "transition entre les problèmes philosophiques auxquels il s'était d'abord consacré et la théorie ethnologique vers laquelle il se tournait depuis quelques années » (Lévi-Strauss 1964b: 182, c'est nous qui soulignons) avait été sans doute plus tranchée. En effet, plutôt qu'une tentative de conjugaison, Marxisme et structuralisme apparaît à double titre comme une tentative de dépassement de l'œuvre de Marx : non seulement Sebag constate que le marxisme s'est trouvé infirmé d'une manière paradoxale par les organisations qui s'en réclament (et d'où il s'était sorti de manière éclatante), mais il arrive jusqu'à exclure « une théorie affirmant le primat de l'économie pour l'ensemble de l'histoire humaine » (1964b : 88). 


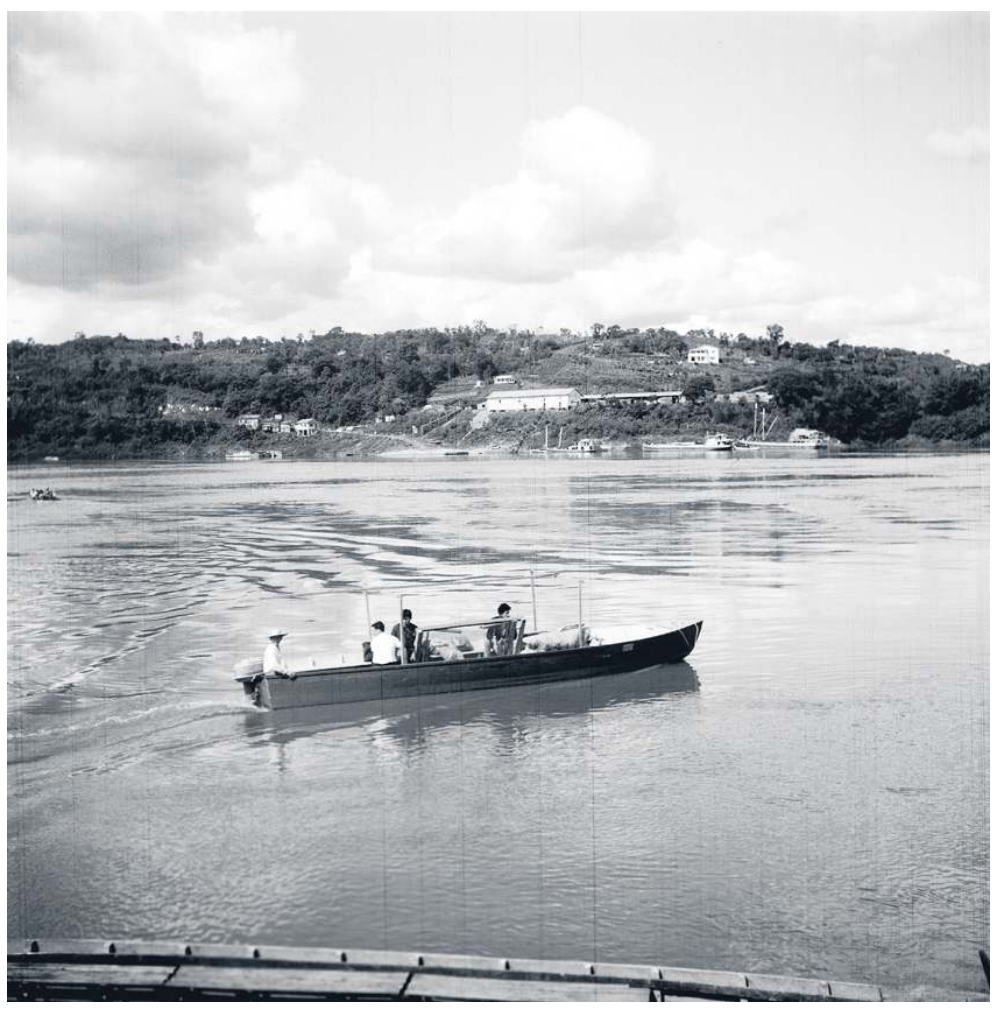

(c) Laboratoire d'anthropologie sociale, fonds Sebag

Le « primat des infrastructures » est certes incontestable, mais dans un sens qui n'est pas pour Sebag celui, restreint, de Marx. Dans L'Invention du monde chez les Indiens pueblos (1971 : 470-471) il se doit de préciser qu'il faut prendre le terme «infrastructure » dans son sens le plus général, car il désigne par là tout substrat géographique, économique et politique. Tout en admettant que les sciences de l'homme ne sont pas encore capables de le prouver, pour Sebag il est clair que « la reconnaissance du primat de l'économique est un jugement d'existence qui appelle une vérification empirique et qu'aucune déduction ne peut fonder " $(1964 b: 88$, n. 1). Comme on le verra, c'est l'étude de la structure et du fonctionnement des systèmes symboliques qui a permis à la linguistique et à l'ethnologie une vérification plus précise des rapports entre idéologie et pensée scientifique, tout en montrant la nécessité de ramener à de justes proportions la portée du marxisme et de ne pas faire découler sa validité de son efficience sur le plan politique. En ce sens, Sebag développe de manière cohérente et originale la pensée de Lévi-Strauss qui, dans Tristes Tropiques (1955), tout en considérant le marxisme, avec la psychanalyse et la géologie, comme une des sources du structuralisme, ne s'empêche pas d'en prendre la mesure afin de donner à l'anthropologie un statut autonome et scientifique. C'est le «marxisme pessimiste " de Lévi-Strauss, puisque les sociétés primitives ne représentent pas la première phase de l'humanité et parce que son mouvement ne procède pas d'un schéma évolutif capable de libérer l'homme de ses servitudes.

6 Le dépassement de la psychanalyse a certainement été plus complexe, d'autant plus que Sebag l'avait pratiquée en patient et que ses consultations semblent avoir été brusquement interrompues quelque temps avant qu'il se donne la mort. 
7 Pour nous qui n'avons pas connu Sebag et qui essayons de comprendre, est-il possible que différents éléments dont se souviennent ses amis (la possibilité que des traumatismes d'enfance aient perduré jusqu'à l'âge d'homme, son absence au moment de la mort tragique de son frère auquel il avait plusieurs fois sauvé la vie, une déception amoureuse dans la complexité des liens mis en jeu par le transfert analytique, la possession d'une arme qu'il avait rapportée du Paraguay et qu'il caressait ostensiblement devant les autres) l'aient perturbé au point qu'il trouve dans la psychanalyse un milieu favorable à la déconstruction de la personne? Mais resurgissent encore d'autres souvenirs. Ses amis, qui habitaient presque tous dans de grandes chambres d'hôtel au cœur de Paris (comme Sebag, qui logeait au 57, rue de Seine, là où Baudelaire avait habité), se rappellent l'atmosphère joyeuse des soirées enfumées consacrées à la belote après la fermeture de la bibliothèque de philosophie de la Sorbonne. Reviennent aussi en mémoire les visites soudaines de François Châtelet qui, ayant eu Sebag comme élève au lycée Carnot de Tunis, l'avait encouragé à partir avec lui pour Paris à l'âge de vingt ans ${ }^{1}$. Ils évoquent encore l'image de Lucien, séducteur par la parole, qui disparaissait, un livre sous le bras, pour improviser quelques jours plus tard des conférences sur de nouveaux thèmes.

Les terrains de Sebag furent courts, mais productifs au plus haut point. La raison en est que l'on ne va jamais sur le terrain «nu et cru ». Sa formation auprès de Lévi-Strauss lui avait permis non seulement de prendre progressivement ses distances, certes qu'intellectuelles, vis-à-vis de sa formation philosophique, mais aussi d'élaborer une stratégie d'enquête dont ses cahiers de terrain fournissent la preuve la plus évidente. D'abord témoignant de l'enthousiasme chaotique qui caractérise souvent l'enquête, puis recopiées d'une calligraphie ronde et mince qui s'étend jusqu'au bord extrême de la page, les notes de Sebag rendent compte d'une rigueur intellectuelle, d'une capacité d'observation et d'un souci de l'essentiel hors du commun.

9 Bien qu'elle privilégie les thèmes ayant fait davantage l'objet de son exercice structural auprès de Lévi-Strauss - la mythologie, le chamanisme ou la guerre - ou qu'elle se réfère à l'autre volet de sa formation - les pathologies mentales ou les rêves -, l'enquête de Sebag ne néglige presque aucun des aspects des deux cultures indiennes étudiées : les AchéGuayaki qui occupent, aujourd'hui encore, la forêt de la zone orientale du Paraguay et les Ayoré-Moros du Chaco paraguayen et bolivien. Ainsi, la culture matérielle et le système de parenté, la chefferie et l'organisation clanique, le cycle de vie et les cérémonies calendaires s'enchaînent en progression ordonnée dans ses cahiers, dans un état d'élaboration semblant parfois déjà prêt pour la publication. Ces notes révèlent une tendance marquée à poser les bonnes questions et une maîtrise extraordinaire de la discipline.

Dans son «Compte rendu de mission chez les Indiens ayorés du Paraguay et de Bolivie ", Sebag (1964c) ne fait aucune mention de son terrain chez les Guayaki. Il y avait pourtant séjourné de février à septembre de cette même année en compagnie de Pierre Clastres, dans le cadre de la mission au Paraguay que Lévi-Strauss avait demandé à Alfred Métraux d'organiser - dans sa note introductive à l'article posthume de Sebag sur «Le chamanisme ayoréo ", Lévi-Strauss (1965) rappelle que Métraux (qui avait contribué à former Sebag), contrairement à son intention déclarée, ne rejoignit jamais cette mission ${ }^{2}$. Or, son terrain chez les Guayaki confirme déjà toutes les qualités que Lévi-Strauss reconnaît à Sebag dans sa préface à L'Invention du monde. Cet ouvrage sur les mythes d'origine des Indiens pueblo avait été conçu dans le cadre du séminaire de celui-ci à l'École pratique des hautes études, et Sebag lui avait remis le manuscrit peu de temps 
avant de se donner la mort. Bien qu'émergeant à l'occasion d'un travail fondé sur des sources documentaires, "puissance de travail, méthode exigeante, logique implacable alliée à une sensibilité frémissante, imagination créatrice» sont les qualités qu'on retrouve dans les deux articles sur les Guayaki que Sebag publia pendant son terrain et à son retour ("Cannibalisme et mort chez les Guayakis (Achén) », en 1963, en collaboration avec Pierre Clastres ${ }^{3}$ et "Analyse des rêves d'une Indienne guayaki», en 1964). Ces qualités apparaissent aussi dans toute une série de matériaux, encore inédits, où la relation entre la formation théorique et le terrain apparaît très étroite. C'est la manière dont les données émergent au cours de la pratique ethnographique qui témoigne souvent de la bonne préparation du chercheur.

11 Il en est de même en ce qui concerne son séjour chez les Ayoré, d'abord à la mission catholique Maria Auxiliadora du haut Paraguay - où les Indiens avaient été transplantés hors de leurs territoires traditionnels du Chaco central, en 1963, un an après l'établissement de relations stables avec les Blancs ${ }^{4}-$, ensuite à Tobité, dans une des missions évangéliques situées dans la partie orientale du département de Santa Cruz, en Bolivie, où le contact avait été établi une quinzaine d'années plus tôt qu'au Paraguay. Les dates et les lieux de ses enquêtes chez les Ayoré du Paraguay posent des problèmes auxquels l'étude de ses cahiers, qui ne contiennent que très rarement des informations chronologiques, n'a pas encore apporté d'explication. Sebag (1964c: 126) écrit avoir séjourné en septembre et en octobre 1963 "dans la mission catholique du Chaco paraguayen ", sans pour autant préciser laquelle, puis du 15 novembre au 15 janvier dans la mission protestante de Tobité (Bolivie) où il dit avoir obtenu les résultats les plus importants. Or, excepté les nombreux contacts sporadiques qui eurent lieu dans le Chaco à partir de la capture en 1956 d'un jeune Ayoré (Ikebi) d'une douzaine d'années par les colons paraguayens, le début de la sédentarisation, due à la décision des Ayoré euxmêmes lorsqu'ils comprirent que sur le plan militaire ils étaient perdants, date de 1962 et eut lieu à Fortín Batista. Par ailleurs, c'est là que l'ethnolinguiste d'origine slovène Branislava Susnik réalisa sa première enquête sur «La lengua de los Ayoweos-Moros » (dont le sous-titre est " Estructura gramatical et fraseario etnográfico »), publiée en 1963, alors que les Ayoré, d'après les remémorations qui en ont été faites, n'auraient été transplantés dans la mission salésienne de Maria Auxiliadora qu'en novembre 1963. Nous avons cependant tendance à croire que la date la plus ancienne notée par Sebag est la date exacte, d'autant qu'elle est confirmée par le témoignage du père salésien Bruno Stella. Dans ses mémoires, celui-ci attribue le suicide de Sebag à une raison clinique comprenant les «crises mystiques » dont il aurait été affecté lors de son bref passage à Maria Auxiliadora. Que Sebag puisse avoir été sensible aux problèmes de l'univers religieux, la qualité de son adhésion au marxisme le montre. Il pensait d'ailleurs à un travail, L'Église de demain, dont il ne reste que la trace du titre dans ses cahiers de terrain (cf. note 20). L'hypothèse d'une "crise mystique " nous paraît, en revanche, très improbable et aussi fantaisiste que celle des Ayoré qui, eux, attribuent le suicide de Sebag - de même que la mort de l'ethnologue argentin Marcelo Bormida ou la maladie mentale d'un de leurs meilleurs informateurs, Samane - au fait qu'il avait voulu qu'on lui raconte des parties de mythes interdites, puyák.

Quelles que soient les dates précises, Sebag est jusqu'à présent un des rares anthropologues à avoir réalisé une expérience de terrain de chaque côté des salines situées à la frontière des deux pays et qui avaient été traditionnellement un lieu de rencontre et d'affrontement entre les différents groupes ayorés avant qu'ils soient 
englobés dans leurs entités nationales respectives. Il est vrai que les informations et la cinquantaine de mythes qu'il a recueillis proviennent en grande partie de son séjour bolivien, surtout grâce à l'aide d'une missionnaire, Violeta Bruchegger ${ }^{5}$, installée depuis longtemps chez les Ayoré et qui connaissait parfaitement leur langue. Cependant, c'est au Paraguay que Sebag dit avoir observé, avant de le faire en Bolivie, le rituel de guérison chamanique décrit dans son article (notamment auprès du chamane Acui, qu'il photographie plusieurs fois à Maria Auxiliadora).

Il faut ajouter à cela au moins trois éléments. D'abord, l'importance de la rencontre avec deux groupes ayorés qui, au Paraguay, venaient d'être contactés et qui faisaient le va-etvient entre la mission et la forêt (aujourd'hui encore des dizaines d'Ayoré du Chaco central n'ont jamais eu de contacts avec la « civilisation des Blancs »). Ensuite, le début de l'élaboration d'un vocabulaire, en tête duquel Sebag note, à côté du terme méprisant « Moros » par lequel les Paraguayens appelaient ces Indiens, le terme « Ayubeo », dont la prononciation exacte est Ayoweo, qui veut dire "personne humaine ». Après quelques années, Ayoweo sera hispanisé en Ayoréo, selon un processus de contamination linguistique qu'en Bolivie Sebag trouvera déjà accompli ${ }^{6}$. Il est intéressant de signaler que dès que l'écoute des sons de cette nouvelle langue se perfectionne, Sebag écrit correctement Ayoweode (qui est le pluriel de Ayoweo). Enfin, c'est de Maria Auxiliadora qu'est issue la quasi-totalité des cinquante-six pièces qu'il rapporte du terrain, pièces que Lévi-Strauss donnera au musée de l'Homme, et dont trente-sept sont accompagnées par des fiches que Sebag lui-même avait rédigées de retour à Paris. Dans ses cahiers, il n'y a aucune trace des annotations originales. Les fiches, rédigées entre les mois de mai et d'octobre 1964, ne correspondent pas à la progression de l'enquête. D'après celles-ci, il n'y a que deux objets qui ont été recueillis à Tobité, en Bolivie, alors que tous les autres l'ont été à Maria Auxiliadora, au Paraguay, sauf « les bâtonnets servant à faire du feu », trouvés à Ingavi, ancienne dénomination de Fortín Capitan Lagerenza. Sebag fait également référence dans ses cahiers à cette localité du Chaco central paraguayen en dressant la liste des deux groupes ayorés qui y étaient présents, alors qu'il ne parle jamais de la mission salésienne de Fortín Batista (ou plus précisément du Fortín Colonel Martínez, ancien Fortín Batista, situé à 39 kilomètres).

14 L'intérêt de ces objets est double. D'une part, ce sont des pièces d'une extrême beauté qui étaient encore toutes utilisées : des bandeaux frontaux en peau de jaguar, des colliers de plumes et autres ornements de guerre, des pierres pour la peinture faciale, des sacs en fibre de caraguatà, des sandales, des baguettes à feu, des pipes, des hochets et des sifflets, des armes. D'autre part, comme ceux recueillis par Branislava Susnik quelques mois plus tôt à Fortín Batista et qui sont conservés au musée ethnographique Andrés-Barbero d'Asunción, ces objets offrent une précieuse opportunité de comparaison avec ceux que les Ayoré fabriquent aujourd'hui, pour les vendre en ville, dans quelques bourgades mennonites de la région ou aux rares visiteurs qui arrivent dans leurs villages. L'étude de ces objets permet de comprendre par quels mécanismes les Ayoré gèrent leurs propres émotions esthétiques vis-à-vis des changements de tous ordres qui ont bouleversé leur culture ${ }^{7}$.

La qualité de cette première mission au Paraguay et en Bolivie n'avait pas échappé à LéviStrauss, qui pensait déjà qu'elle serait suivie d'une seconde dès que Sebag aurait soutenu sa thèse (en juillet 1965), dont il avait clairement exposé le propos scientifique dans son compte rendu de mission et dans quelques séminaires. À partir du 15 janvier, il aurait dû tenir, avec Clastres, les conférences de la fondation Loubat que le Collège de France leur 
avait confiées. La perspective dans laquelle il affirme vouloir traiter le matériel recueilli chez les Ayoré (les mythes surtout) est celle de la comparaison avec les autres sociétés du $\mathrm{Chaco}^{8}$ (dont les connaissances étaient suffisamment étendues), alors que dans L'Invention du monde il avait appliqué la méthode structurale à l'analyse d'une seule classe de mythes (ceux d'émergence) d'une unique société, celle des Pueblo orientaux du groupe linguistique keresan, en limitant la comparaison aux mythes du même type des Pueblo occidentaux (Zuni et Hopi ${ }^{9}$ ). C'est encore dans la préface à L'Invention du monde que LéviStrauss rappelle avoir demandé en 1960 à Sebag - que Jean-Claude Gardin lui avait présenté - d'animer une équipe destinée à étudier la mythologie des Pueblo. Lévi-Strauss (1952-1953, 1953-1954) avait lui-même commencé ce travail de 1951 à 1954, avant de choisir définitivement quelques années plus tard, chez les Bororo du Brésil central, les mythes qui lui servirent de fil conducteur pour mettre sa méthode à l'épreuve. Sebag y travailla pendant trois ans, avec l'aide de Simone Dreyfus, Jacqueline Bolens, Judith Lacan et Pierre Smith, tout en sachant que cette mythologie devait être étudiée " en elle-même et pour elle-même » avant d'être intégrée dans un système plus vaste.

Le programme de Sebag prévoyait donc le passage de l'analyse du mythe dans un seul ensemble culturel à celle des mécanismes de la pensée mythique dans un ensemble géographique régional diversifié du point de vue ethnique (le Chaco), suivant ainsi un parcours scientifique que Lévi-Strauss venait d'inaugurer dans Le Cru et le Cuit (1964a). Il y a là quelques points de méthode à souligner.

On peut distinguer dans l'analyse structurale des mythes plusieurs niveaux d'investigation relevant tous de la même architectonique. Il s'agit avant tout de pouvoir faire apparaitre l'ensemble paradigmatique du déroulement d'une unique chaîne syntagmatique. Cette procédure est conduite de telle sorte que l'on puisse traduire la succession des événements présents dans un mythe - sa mélodie, pour utiliser la métaphore lévi-straussienne de la partition d'orchestre - en une série de "colonnes » de relations analogues - dans les mêmes termes musicaux, l'harmonie. C'est la méthode que Lévi-Strauss (1958 : 227-255) met à l'épreuve pour la première fois, en 1955 (éd. anglaise), dans son article " La structure des mythes », en analysant le mythe d'ÆEdipe.

Tout d'abord, le mythe est décomposé en unités minimales isolées au niveau de la phrase (les grosses unités constitutives, ou mythèmes), dont la signification est double: la première se référant strictement aux autres éléments du récit, la seconde au contexte ethnographique. Ensuite, on acquiert la possibilité de mettre en évidence une matrice achronique de termes opposés et corrélés les uns aux autres à travers les affinités thématiques qu'ils entretiennent entre eux. Enfin, on en vient à la recherche d'analogies à l'intérieur de chaque colonne et d'inversions entre les différentes colonnes.

Un deuxième niveau possible d'analyse structurale est celui qui prend en considération les mythes d'un genre particulier d'une seule société ou d'un seul ensemble culturel. Un autre niveau encore est celui que Lévi-Strauss a exploré dans les Mythologiques à l'échelle du continent américain. En considérant le mythe comme l'ensemble de ses variantes, dont les rapports d'analogie et d'inversion (leur donnant la forme d'un groupe de permutations) sont analysés par la logique transformationnelle qui préside à la formule canonique $^{10}$, Lévi-Strauss a pu non seulement montrer que les variations entre les mythes d'un même type sont liées à la relation différente que chaque groupe ethnique entretient avec l'environnement, mais encore déceler des propriétés fondamentales de la pensée mythique. Ce qui ouvre vers un quatrième niveau, qui se cache derrière la question de l'existence d'une pluralité de mythologies qui contribuerait à caractériser les cultures ou 
d'une mythologie unique qui se rapprocherait d'une production unitaire de l'esprit humain.

Apparemment la plus simple, car elle ne s'applique qu'à un seul récit, la procédure qu'illustre le mythe d'ÆEdipe sollicite à vrai dire tous les principes de méthode, élaborés à partir de la linguistique saussurienne et de la phonologie, qui sont à la base de l'analyse structurale. Dans l'article de Lévi-Strauss, il n'y a pas de solution de continuité entre l'analyse du mythe d'ÆEdipe et l'énonciation de la formule canonique suite à l'étude du mythe zuni d'émergence. N'en déplaise à ceux qui poursuivent une perspective néorelativiste en anthropologie, pour qui la pensée amérindienne se déploie selon des modes irréductibles à ceux d'autres sociétés humaines - un de ces modes serait la négation de l'altérité découlant de la prédation ${ }^{11}$-, Lévi-Strauss affirme avoir choisi le mythe d'ÆEdipe comme " premier exemple » de sa démonstration non seulement parce qu'il est connu de tous, mais encore en raison des «analogies remarquables qui existent entre certains aspects de la pensée grecque archaïque et celle des Indiens pueblo ». Il s'agit notamment $\mathrm{du}$ rapprochement entre les pouvoirs "énigmatiques» du sphinx et ceux de deux personnages de la mythologie nord-américaine - qui en réalité « n'en forment sans doute qu'un seul ", comme le précise Lévi-Strauss : la vieille sorcière et la mère phallique, toutes les deux nécessaires au succès du héros. De même, on retrouvera la difficulté à marcher droit (qui exprime dans le mythe d'Ædipe la permanence de l'autochtonie humaine) dans les mythes des Pueblo (et des Kwakiutl) où les êtres qui participent au mythe d'émergence sont boiteux.

Le chamane Acui (clan Possoahá) exécute un chant ; à gauche, Lucien Sebag. Maria Auxiliadora, septembre-novembre 1963.

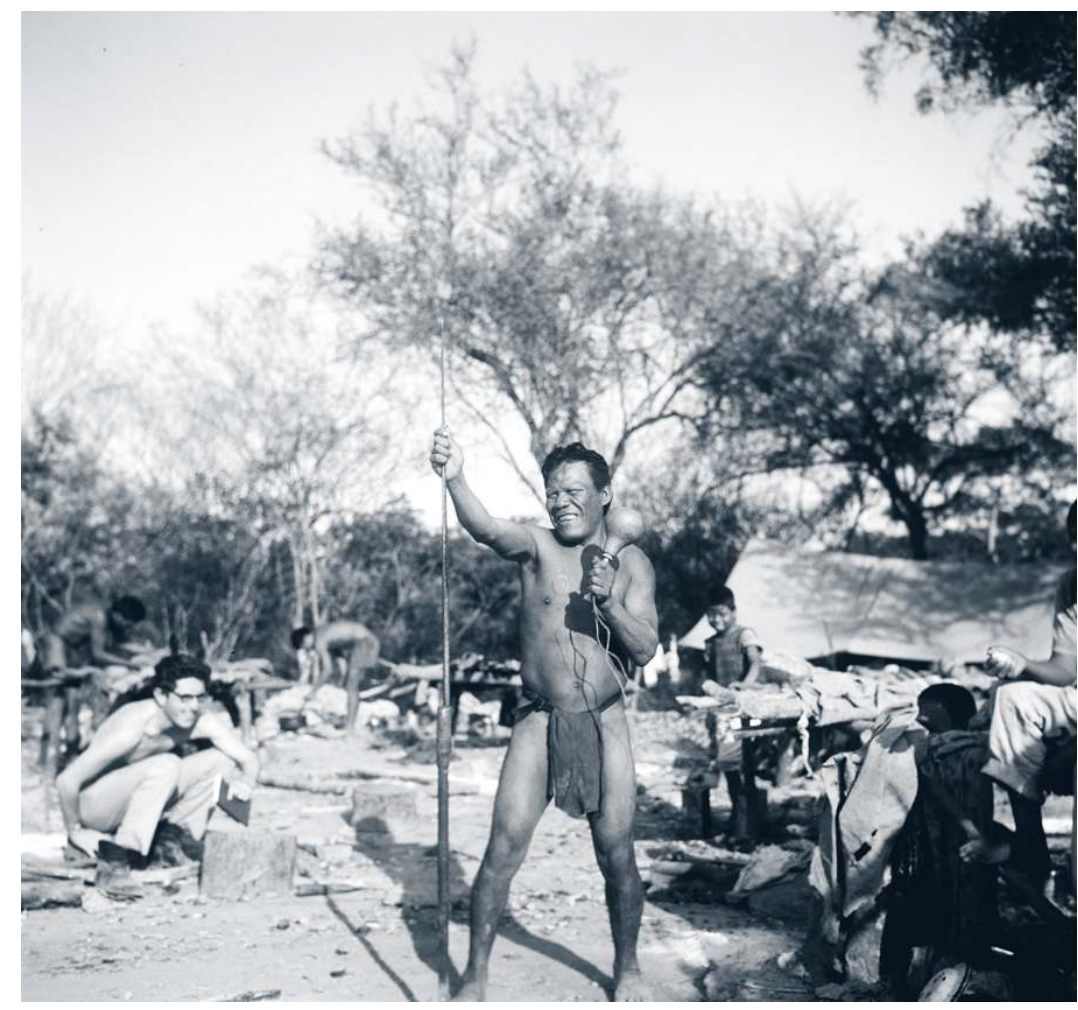

(C) Laboratoire d'anthropologie sociale, fonds Sebag 
21 L'unité des matériaux américains ne justifie donc pas l'idée d'un monde à part, où des principes uniques seraient en vigueur. Seule la connaissance de ces matériaux a conduit Lévi-Strauss à les choisir comme le contexte où énoncer la formule canonique. Mais il avait envisagé implicitement la possible application de celle-ci également au mythe d'৫Edipe, pourvu qu'on ne se limite pas aux variantes thébaines. Puisque ce mythe se compose, comme tout autre, de l'ensemble de ses variantes, leur prise en charge par l'analyse structurale se devait de donner un ensemble tridimensionnel, dont la lecture n'aurait plus été seulement de gauche à droite et de haut en bas (comme dans le premier exemple), mais aussi d'avant en arrière (ou vice-versa). Le recours au symbolisme d'inspiration mathématique, dont la formule canonique n'est, selon Lévi-Strauss, qu'une indication approximative, découle en effet du passage à des systèmes pluridimensionnels. Produits par la multiplicité des variantes dont le mythe se compose, ces systèmes ne peuvent pas être maîtrisés par les méthodes empiriques traditionnelles ${ }^{12}$.

Le choix obligé du deuxième niveau n'empêche pas Sebag de tenir compte, dans L'Invention du monde, de la procédure large que Lévi-Strauss avait élaborée dans l'article sur la structure des mythes ${ }^{13}$ puis mise en œuvre dans Le Cru et le Cuit. À ce propos, il est significatif qu'il fasse référence, par analogies et différences, aux mythes ayorés et guayakis, même si ce rapprochement n'est utilisé que pour préciser quelques questions de fond. Différents par leur forme comme par leur contenu, les mythes ayorés traitent du même problème que ceux des Indiens pueblo, c'est-à-dire de la relation de l'esprit au monde ${ }^{14}$. L'esprit privilégie certains traits plutôt que d'autres, mais il se démarque de toute diversité historique et sociale en nous dévoilant, conformément à ses propriétés, le système inconscient qui régit la chaîne syntagmatique et certaines structures permanentes de l'expérience humaine. Pour Sebag, c'est ici que réside la portée philosophique de l'analyse structurale aussi bien que cette reprise herméneutique du mythe à travers laquelle le sujet interprétant se transforme. «La reprise herméneutique des mythes, écrit-il, est constitutive du rapport du sujet au mythe dont il reconnaît la législation.» (1971: 476) Sebag illustre cette position théorique par un mythe guayaki concernant un geste destructeur auquel les Indiens font référence pour stigmatiser tout acte irresponsable : «Les Guayaki content que les hommes sortant du monde souterrain se trouvèrent dans un univers où il faisait toujours jour. La nuit, qu'ils considèrent comme très dangereuse, était enfermée dans une immense marmite que possédait une des divinités primordiales. Mais un jeune homme non encore initié frappa la marmite avec le bois de son arc et la brisa, libérant toutes les puissances nocturnes » (ibid.). Dans le discours de Sebag, la fidélité au mythe est poussée à l'extrême et passe tout d'abord par le texte et une implication personnelle caractérisant à la fois celui qui le raconte aussi bien que l'anthropologue qui l'interprète.

D'autres aspects témoignent de cette fidélité. En soulignant le caractère expérimental de son analyse, Sebag veut, d'une part, rendre compte d'un parcours qui s'intègre dans l'entreprise plus vaste menée par Lévi-Strauss, d'autre part représenter le caractère provisoire de ses résultats, qui découlent, selon ses propres termes, de l'état « imparfait » des mythes étudiés. La nature de ces mythes (leur caractère fragmentaire ou leur longueur excessive) ne lui permettant pas de les fractionner en unités élémentaires, Sebag suit une procédure que Lévi-Strauss adopte parfois, c'est-à-dire la division du récit en séquences d'une taille permettant de faire droit au commentaire de l'ethnologue.

24 À la fin de son parcours, les procédures d'analyse des mythes mises en œuvre par Sebag ne retiennent presque rien de sa formation philosophique initiale. En revanche, il 
reconnaît, tout en commençant à le développer de manière originale, le modèle linguistique qui est à la base de l'anthropologie structurale et dont les principes avaient été énoncés par Ferdinand de Saussure dans son Cours de linguistique générale (l'opposition entre langue et parole, métaphore et métonymie, synchronie et diachronie ou entre la succession syntagmatique et le système paradigmatique).

En appliquant ces concepts à l'analyse des mythes, Sebag sera porté à tenir compte de la spécificité d'un objet autre que la langue, et cela en accord avec l'approche dessinée par Lévi-Strauss qui, dans son article fondateur "L'analyse structurale en linguistique et en anthropologie ", met en garde contre une fidélité trop littérale à la méthode du linguiste, étant donné, par exemple, «la différence très profonde qui existe entre le tableau des phonèmes d'une langue et le tableau des termes de parenté d'une société » (1945). Bien qu'il ne soit pas question ici de mythes, car c'est l'atome de parenté qui est énoncé en conclusion de ces présupposés théoriques, la manière dont l'anthropologie structurale transposera à partir de ce moment la méthode phonologique à ses objets d'étude est désormais clairement exprimée ${ }^{15}$. Il s'agit, en quelque sorte, de l'application d'une logique de distinction que Benveniste aurait clairement formulée quelques années plus tard en reconnaissant que les signes ne fonctionnent pas tous de la même manière ni ne dépendent tous d'un système unique : «On devra constituer plusieurs systèmes de signes, et entre ces systèmes, expliciter un rapport de différence et d'analogie ${ }^{16}$."

Inscrit dans ce courant de pensée, Sebag (qui avait suivi les cours d'André Martinet à la Sorbonne) propose une analyse des mythes qui perfectionne les outils de l'anthropologie structurale, en toute cohérence avec l'esprit des principes établis par Saussure (ce qui lui a permis de profiter des progrès de la linguistique) et les caractéristiques propres à l'objet étudié. C'est dans un double sens que Sebag marque le discours mythique par rapport au langage, aussi bien au niveau de la parole qu'au niveau de la langue, pourrait-on dire en termes saussuriens. En analysant les différents types de communication auxquels les sujets qui interviennent dans le processus de création du mythe acoma ont recours, Sebag (1971: 50) reconnait, par exemple, quatre usages de la parole par lesquels ces sujets visent des fins distinctes : la parole créatrice, informatrice, interrogative et rituelle. Ces modes d'activité linguistique s'articulent les uns aux autres, mais il n'y a que les personnages centraux du mythe, les deux sœurs démiurges Iatiku et Nautsiti, qui peuvent les utiliser tous les quatre. D'autres précisions, relevant du domaine de la langue, éloignent le mythe de manière encore plus tranchante des termes linguistiques dont il se sert. Comme le langage, le discours mythique trouve la matière qui lui sert de support au moyen d'un choix arbitraire dans le milieu naturel et humain, mais il doit s'en détacher pour déployer toute sa force créatrice par rapport à la réalité sociale dont il surgit. 


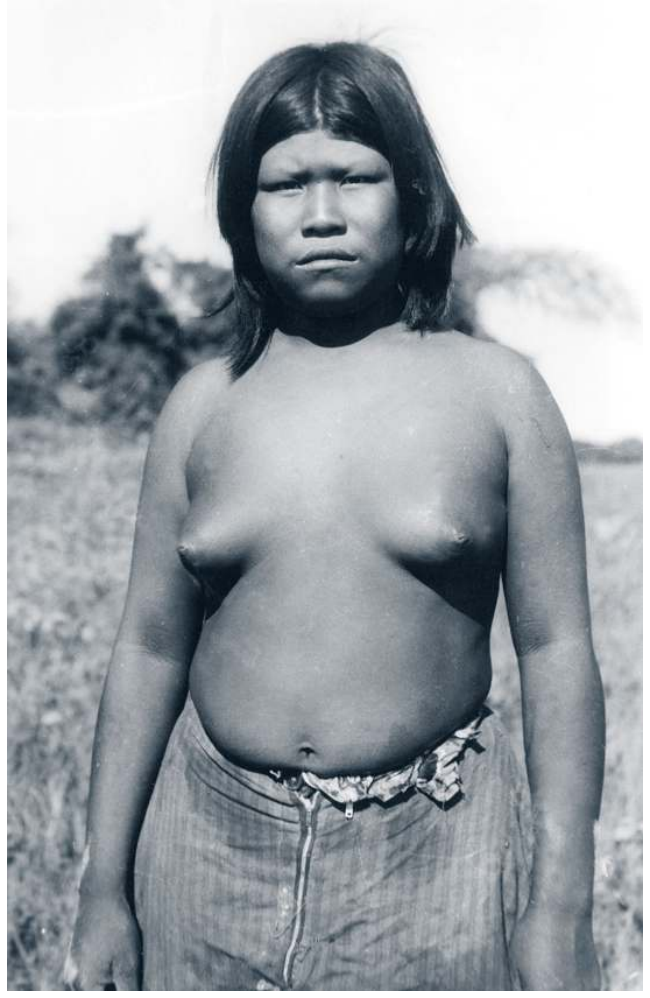

(c) Laboratoire d'anthropologie sociale, fonds Sebag

On se trouve là au cœur de la fonction de soudure qui est propre au mythe car il déplace sur un plan symbolique les contradictions inconciliables de l'existence humaine. Ce transfert oppose les termes antinomiques dans un cadre formel qui les rend signifiants et, tout en montrant leur complémentarité par l'apparition d'un troisième terme médiateur - auquel un autre terme s'oppose et ainsi de suite au moyen de médiations progressives potentiellement infinies -, rejoint le mode opérateur de l'intellect et la logique sousjacente à l'organisation sociale. L'analyse structurale des mythes marque sa différence par rapport à la linguistique structurale en ce qu'elle reconnaît comme trait distinctif de tout discours mythique le double niveau de signification des mythèmes : par la valeur qu'ils assument d'une part dans l'histoire racontée par le mythe, de l'autre dans le contexte ethnographique. Sebag ne pouvait pas exprimer cette différence de manière plus claire quant aux termes mis en relation par le mythe : «Ces termes, quels sont-ils? Le mythe suppose la langue. Il combine des signes, unions d'un signifiant acoustique et d'un signifié, qui lui sont fournis par la société où il s'élabore, mais c'est pour les soumettre à un traitement particulier " (ibid. : 455-456). Tout en retenant l'utilisation qu'en avait faite Lévi-Strauss dans Anthropologie structurale (1958: 101), d'une part Sebag (1964b : 95-96) tient compte, sans les citer, des critiques de Benveniste (et après lui de Jakobson) conduisant à réduire la marge d'arbitraire du signe linguistique, d'autre part il confirme que, du point de vue structural et non historique, le principe saussurien reste inchangé. C'est par rapport à cet arbitraire du signe linguistique que le caractère spécifique de la parole mythique se précise. La contingence du rapport entre les deux faces du signe est de même nature que la communication humaine et relève « de la structure de tout système sémiologique qui est à la fois structuration unitaire de deux 
plans hétérogènes de la réalité dans laquelle les éléments sont puisés et définition différentielle de ces éléments qui ne sont porteurs d'information que dans la mesure où ils s'opposent à des unités du même ordre " (ibid.: 96). En revanche, tout système symbolique, et notamment tout discours mythique, est communiqué par un message supposant une codification supplémentaire par rapport à la langue. Concernant à la fois la «culturalisation» de la nature et la spécificité du discours mythique, ces précisions seront reprises dans L'Invention du monde : «Dans la langue, le rapport entre signifiant et signifié est arbitraire, ce qui ne veut pas dire immotivé. Il n'y a pas de raisons de principe pour que le signifié "bœuf" soit rendu par le signifiant (böf), et la meilleure preuve c'est que, dans une autre langue, un son différent pourra recouvrir le même concept. Le mythe par contre utilise des unités déjà pourvues d'un sens. Ferdinand de Saussure refusait à la langue la possibilité d'inclure des termes positifs. Elle n'est rien d'autre qu'un "système de différences". La formule ne vaut pas pour le mythe, puisque chacune de ses phrases possède un contenu avant son apparition dans un texte mythique. » $(1971: 456)$ Le travail du mythe se caractérise en effet par ce va-et-vient que ses unités minimales permettent de réaliser entre le contexte ethnographique d'où elles surgissent et le récit qui les met en forme par paquets de relations. C'est ainsi qu'elles prennent la double signification dont nous avons parlé et dont l'analyste ne peut se passer.

L'autre principe, souvent oublié, qui est à l'œuvre dans les mythes et dont Sebag tire largement profit, est celui de la redondance, c'est-à-dire la répétition d'une même séquence qui rend manifeste leur structure panchronique (ou synchro-diachronique ${ }^{17}$ ). Sebag (1964a: 2183) attribue à la redondance une signification importante aussi dans l'analyse des rêves, qu'il s'agisse de la récurrence de thèmes ou de personnages mettant en évidence la manière dont s'articule une constellation familiale ou bien de la présence décroissante de certaines données, ce qui montre, à la fois, l'existence de tournants psychologiques et l'existence d'un procès. Avec les associations et les souvenirs d'enfance du rêveur, la dimension temporelle se révèle, aux yeux de Sebag, un élément indispensable à l'intelligence des rêves, pourvu que l'on considère la production onirique comme formant un seul ensemble. L'adoption de ce principe de l'analyse structurale des mythes permet que les rêves d'un individu puissent se commenter et s'éclairer les uns les autres au fil du temps. Ils sont, en définitive, autant de variantes d'un seul rêve.

Il est vrai que Sebag n'a pas eu le temps d'élaborer une pensée sur le mythe qui ne soit pas calquée sur les découvertes de Lévi-Strauss. Bien qu'encore à l'état d'ébauche dans L'Invention $d u$ monde, sa contribution est cependant déjà très originale sur plusieurs points. En accentuant, par exemple, le caractère sélectif du mythe, Sebag croise deux exigences qui doivent être toujours présentes à l'esprit de l'anthropologue. D'une part, il reconnaît que le mythe opère un choix à travers la multiplicité des significations dont les êtres et les actions sont chargés; d'autre part, il insiste sur la nécessité d'un recours exclusif aux catégories indigènes, ce qui est à l'origine de la valorisation du contexte ethnographique. «Entre toutes les classes possibles dont un terme lexical quelconque peut relever - une sauterelle est un insecte, un animal, un être vivant -, [le mythe] choisit certaines d'entres elles qui constitueront le véritable signifié des unités linguistiques qui apparaissent au cœur du récit. [...] L'analyste se doit donc de déterminer, entre toutes celles qui seraient acceptables, la classe pertinente. Toute utilisation de nos propres classes sémantiques doit être exclue, et seul le découpage opéré par la culture étudiée peut entrer en ligne de compte. En ce sens, la connaissance du contexte ethnographique 
est indispensable à toute analyse mythique, puisqu'elle lui fournira les catégories entre lesquelles un choix pourra être opéré » (1971 : 456-457).

De cette procédure découle un autre aspect novateur de la réflexion de Sebag, qui est la recherche d'un système à plusieurs dimensions pouvant rendre compte de la multiplicité de classes paradigmatiques dans lesquelles les êtres et les actions présents dans le mythe sont inscrits. Cela pour permettre de mieux comprendre à la fois l'apparition de chaque élément dans la chaîne syntagmatique comme représentant de sa classe et l'une des fonctions du mythe par rapport à la vie sociale. «L'ensemble des activités humaines, des institutions, des êtres qui peuplent l'univers s'organisent les uns par rapports aux autres suivant un nombre fini de catégories. Le mythe fournit donc une grille logique suffisamment générale pour coder tout ce qui est et permettre aux individus et aux groupes de situer leurs actions dans un monde ordonné » (ibid. : 464).

Une autre suggestion, que Sebag (ibid. : 456) esquisse à peine et encore dans le cadre d'une utilisation productive de la linguistique structurale, concerne justement la formule canonique du mythe ${ }^{18}$. En partant de la réalité à deux faces qu'est le signe linguistique, il le considère comme le plan d'expression du discours mythique, tout en proposant de réécrire la formule canonique au moyen de la distinction introduite par Louis Hjelmslev des deux plans de l'expression (E) et du contenu (C), correspondant respectivement au signifiant et au signifié, dont la relation $(\mathrm{R})$ définit le signe linguistique :

(ERC) $\mathrm{RC}_{1}$

$\mathrm{E}_{1}$

32 La seule explication que Sebag donne de sa formule concerne l'analyse des matériaux mythiques qu'il est en train d'utiliser ${ }^{19}$. Il en est de même pour une deuxième variante de cette formule, par laquelle il exprime le "vaste système d'équivalence entre plans distincts de la réalité » que le mythe permet de réaliser :

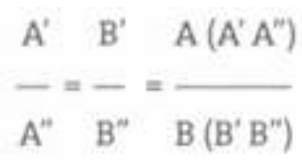

Le discours de Sebag est au contraire très clair. La pensée mythique (dont la procédure logique est révélée par l'analyse structurale) soumet les éléments qu'elle puise dans la réalité à une sorte d'« alchimie » (que Sebag oppose à la théorie du reflet) « qui lui permet de surmonter un certain nombre de contradictions qui, sur le plan réel, sont difficilement solubles » (ibid. : 464).

Une dernière modification de la formule, aussi difficile à saisir que celles qui la précèdent, est proposée par Sebag pour rendre compte du paradoxe qui est propre à tout mythe, c'est-à-dire le fait que « d'une part, les êtres qui interviennent dans le récit ne sont que le signifiant contingent d'un signifié qui se situe à un autre niveau; d'autre part, c'est l'histoire de ces êtres que le mythe raconte» (ibid. : 483-484). Le message du mythe (qui résiste par sa nature même aux substitutions lexicales) se développe donc simultanément sur plusieurs plans, dont l'un est constitué par le matériel signifiant lui-même. C'est ce rapport entre l'aspect sensible du mythe et la logique qui le transcende qui est exprimé par cette nouvelle (et dernière) variante de la formule canonique :

$$
\mathrm{E}_{1} \text { (e R c) R C } 1 \text { (e R c) }
$$

Un ultime aspect significatif de la trajectoire intellectuelle de Sebag concerne ses rapports avec la psychanalyse. Il est possible de les retracer à partir de l'article « Analyse des rêves d'une Indienne guayaki " (1964a) et des «Conclusions méthodologiques » à 
L'Invention du monde. Fortement imprégné par les théories de Freud et de Lacan, Sebag nous présente la relation avec Baipurangi, la jeune Aché qui lui raconta ses rêves chaque matin durant trois mois, comme celle qui s'établit entre un psychanalyste et son patient. $\mathrm{Au}$ «transfert affectif» que Baipurangi opère sur lui, et qui la conduit à un moment donné (le rêve $n^{\circ} 8,12$ dans ses notes de terrain) jusqu'à évoquer des relations sexuelles avec lui et à lui attribuer la responsabilité de "nommer ce désir et de l'accomplir », Sebag oppose une « neutralité affective » qui finit par provoquer le tarissement de cette source. En «rêvant pour Sebag ", Baipurangi met donc en jeu des "désirs plus secrets » que le désir répété de rêver; des désirs que seule une connaissance approfondie du contexte ethnographique permet de décrypter (parmi lesquels, sans aucun doute, le désir de maternité de cette femme qui jusqu'alors n'a pas eu d'enfant). Dans cette perspective, Sebag vise la possibilité d'une "fécondation réciproque " entre la psychanalyse et l'ethnologie, mais à la condition que leur spécificité soit respectée : l'irréductibilité de l'expérience psychanalytique et la critique radicale énoncée par les ethnologues des « extrapolations auxquelles donnaient lieu les "sociétés primitives" » (ibid. : 2237) dans la démarche des psychanalystes ${ }^{20}$.

Dans cet article, le problème des rapports entre les deux disciplines reste ouvert et laisse entrevoir, notamment, les contours de la contribution que Sebag aurait pu donner à l'analyse des relations entre le mythe et le rêve. Pareillement, dans Marxisme et structuralisme Sebag défend la psychanalyse du fait de la propension qu'ont d'autres sciences à la dissoudre dans leurs problématiques. D'une part, il valorise les effets spécifiques de la psychanalyse sur les sujets névrosés (effets dus à l'originalité de cette science ainsi qu'à ses constructions théoriques). Il redimensionne, d'autre part, la critique anthropologique formulée par Lévi-Strauss (1958 : 205-226) dans son article "L'efficacité symbolique ». Selon Sebag, l'interprétation du rituel chamanique cuna, qui consiste à ramener les difficultés de certains accouchements à un ordre mythique qui les outrepasse, tout en faisant jouer une fonction thérapeutique au langage ne contient pas une critique véritable. Il s'agit plutôt «d'une reproblématisation de la psychanalyse à partir d'expériences qui, à bien des égards, peuvent formellement s'en rapprocher " (1964b : 225, n. 1). Pour Sebag, autant que l'ethnologie, la psychanalyse fait encore partie des sciences qui cherchent à intégrer l'opposition entre le moi et l'autre dans le système plus vaste dont ils résultent et dont ils ne sont qu'une des transformations possibles: "L'Homme ne se dévoile qu'en dialoguant avec ce qui n'est pas lui et qui, pourtant, peut apparaître comme son double » (ibid.: 209). Émergent alors les similitudes que les deux sciences cherchent à mettre en lumière entre l'homme occidental et le primitif, entre l'homme normal et le névrosé (rejoignant ainsi la psychologie de l'enfant et la psychologie animale qui, elles, cherchent à comprendre la forme humaine adulte en l'opposant à l'animal et à l'enfant).

Dans L'Invention du monde, au contraire, les critiques de Sebag à l'égard de la psychanalyse ne laissent aucune place à la conciliation. Il la considère comme étant l'une de ces théories non structurales qui cherchent à introduire dans les mythes une cohérence externe (à l'instar des théories historiques et psychologiques). Celles-ci ne retiennent du texte à déchiffrer que "ce qui est conforme au sens proposé » et font ainsi le partage entre ce qui est censé être important et ce qui est censé ne pas l'être. 


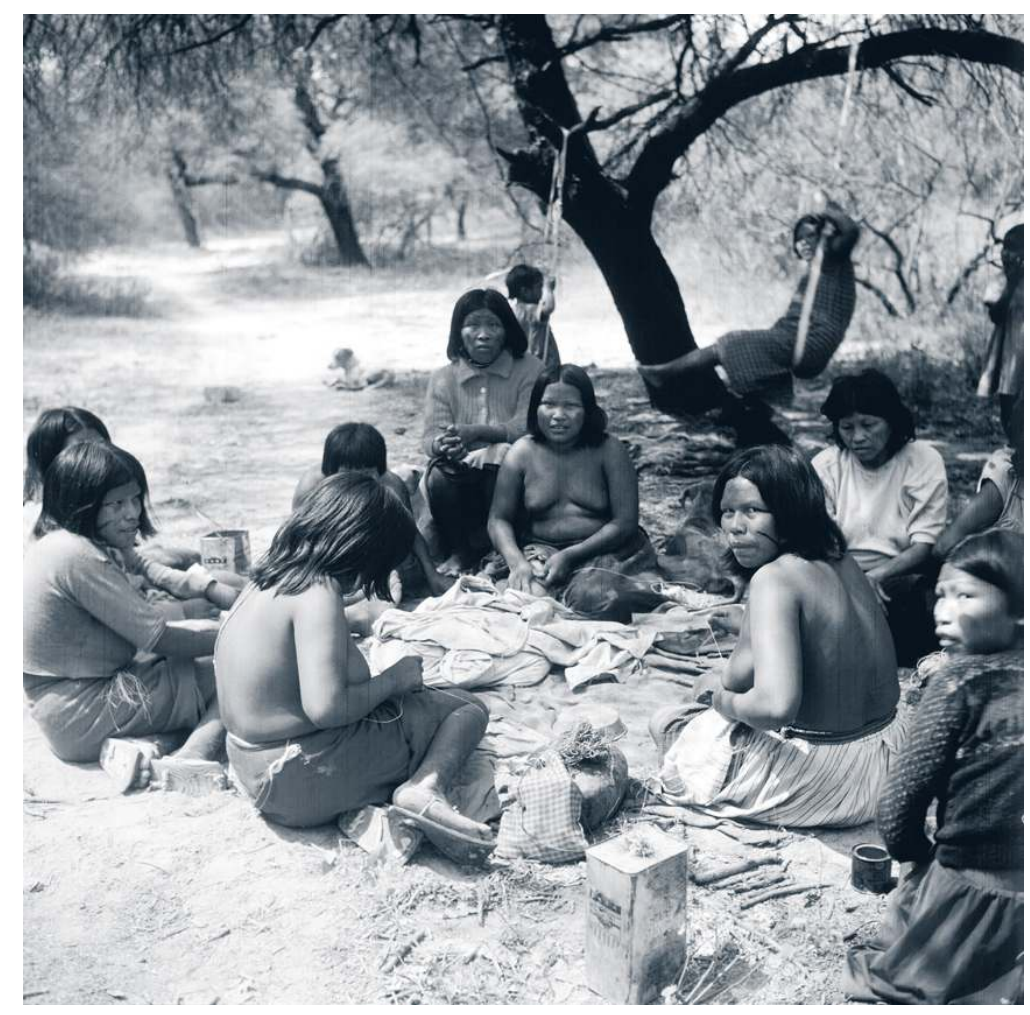

(c) Laboratoire d'anthropologie sociale, fonds Sebag

En essayant de dissiper l'absurdité apparente du mythe par cette cohérence externe qui voudrait l'expliquer d'un seul coup et dans son ensemble, certaines interprétations le confondent avec la théorie de ses origines ${ }^{21}$. La psychanalyse freudienne, notamment, ne voit dans le mythe qu'une opportunité de représenter les conflits œdipiens qui sont à la base de la subjectivité, alors que la variante jungienne s'attache à rechercher «les archétypes intemporels qui sont déposés dans l'âme et lui donnent son épaisseur » (1971: 454). Pour montrer comment les explications extrinsèques finissent par faire éclater le mythe en ne jugeant pertinents que quelques fragments sélectionnés a priori, Sebag reprend les critiques de Melville et Francis Herskovitz de l'analyse d'Otto Rank des mythes centrés sur le personnage d'œedipe meurtrier de son père. La cause initiale du drame est déplacée de l'un à l'autre personnage, puisqu'il n'y a pas seulement le sentiment de haine du fils vis-à-vis de son père - coupable, à ses yeux, de l'avoir exilé ou chassé - mais aussi et d'abord la peur du père d'être détrôné par son fils. Des éléments «secondaires » du récit deviennent ainsi intelligibles, tout en permettant de récupérer le thème de la transmission de la souveraineté que l'adoption exclusive du modèle de relation père-fils proposé par Freud dans son commentaire au mythe d'œdipe avait laissé dans l'ombre.

Sebag ne semble pas avoir choisi cet exemple au hasard. Il avait certainement à l'esprit à la fois la critique formulée par Lévi-Strauss dans Les Structures élémentaires de la parenté (1949) de l'explication freudienne de la prohibition de l'inceste et l'énonciation de la méthode structurale précisément dans l'article où Lévi-Strauss analyse le mythe d'Ædipe. On est là bien loin d'une quelconque tentative de conciliation entre les deux perspectives, psychanalytique et structurale, qui avait été opérée dans l'« Analyse des rêves " et 
Marxisme et structuralisme. En l'espace de quelques mois après son retour du terrain, la pensée de Sebag concernant le rapport entre la psychanalyse et la théorie structurale du mythe avait sensiblement évolué. Les "Conclusions méthodologiques » de L'Invention du monde - ajoutées à la fin d'un texte en grande partie déjà rédigé et dont des extraits ont été publiés après sa mort - en constituent sans doute la preuve la plus évidente ${ }^{22}$.

La manière de procéder de la psychanalyse se situe désormais pour Sebag « aux antipodes de la pensée mythique » et il expliquera ainsi pourquoi l'abord structuraliste « ne peut ne pas s'opposer " aux autres théories d'analyse du mythe: "Celles-ci se préoccupent d'emblée de mettre en rapport les mythes avec autre chose que lui-même ; elles divergent dans la mesure où elles ne choisissent pas le même pôle de référence. Par contre, la première méthode cherche d'abord à rendre compte du texte qui lui est proposé. » Tout en admettant que le mythe puisse relever d'une interprétation psychanalytique, Sebag juge prioritaire d'en dégager le contenu qui est le sien "par des procédés qui n'impliquent pas des présuppositions sur la nature du message déchiffré » (1971: 455).

Les critiques de Sebag rejoignent enfin sur le point le plus important celles que LéviStrauss avait adressées à Freud dans Les Structures élémentaires de la parenté à propos du passage de la nature à la culture, et qu'il affinera quelques années plus tard dans La Potière jalouse (1985). En expliquant pourquoi le structuralisme ne peut pas ne pas s'opposer aux théories historiques, psychologiques ou psychanalytiques, Sebag se place résolument dans les plis de la pensée mythique dont il écrit qu'elle "établit des équivalences multiples entre plans distincts de la réalité naturelle, psychologique, sociale. Elle construit un vaste système de correspondances qui lui permet d'ordonner le monde en révélant une logique sous-jacente à ses différents niveaux. Déclarer qu'un seul de ces plans est significatif revient à faire disparaitre le mythe » $(1971: 455)$. «Le mythe est un discours », écrit encore Sebag. Le discours du mythe est encore loin d'avoir disparu.

\section{BIBLIOGRAPHIE}

BERNAND-MUÑOZ, Carmen

1977 Les Ayoré du Chaco septentrional. Étude critique à partir des notes de Lucien Sebag. Paris-La Haye, Mouton.

DARMON, Jean-Pierre

1966 « Pour Lucien Sebag. In memoriam ». Tunis, La Presse.

LÉVI-STRAUSS, Claude

1945 « L'analyse structurale en linguistique et en anthropologie », Word. Journal of the Linguistic Circle of New York I (1) : 33-53.

1949 Les Structures élémentaires de la parenté. Paris, Plon.

1952-1953 « Recherches de mythologie américaine (1) », Annuaire de l'École pratique des hautes études, Section des sciences religieuses : 19-21. 
1953-1954 « Recherches de mythologie américaine (2) », Annuaire de l'École pratique des hautes études, Section des sciences religieuses : 27-29.

1955 Tristes Tropiques. Paris, Plon.

1958 Anthropologie structurale. Paris, Plon.

1958-1959 « La geste d'Asdiwal », Annuaire de l'École pratique des hautes études. Section des sciences religieuses : 3-43 (repris in Anthropologie structurale II. Paris, Plon, 1973 : 175-233).

1964a Mythologiques I. Le Cru et le Cuit. Paris, Plon.

$1964 b$ « Lucien Sebag », Journal de la Société des américanistes 53 : 182-183.

1965 « Présentation » à Lucien Sebag, « Le chamanisme ayoréo ", L'Homme V (1), janvier-mars : 7-32 ; (2), avril-juin : 92-122.

1971 « Préface » à Lucien Sebag, L'Invention du monde chez les Indiens pueblos. Paris, Maspero : I-III. 1984 Paroles données. Paris, Plon.

1985 La Potière jalouse. Paris, Plon.

1991 Histoire de Lynx. Paris, Plon.

SEBAG, Lucien

1964 (en collaboration avec Pierre Clastres) « Cannibalisme et mort chez les Guayakis (Achén) », Revista do Museu Paulista n.s., XIV : 174-181.

1964a « Analyse des rêves d'une Indienne guayaki », Les Temps modernes 217 : 2181-2237.

1964b Marxisme et structuralisme. Paris, Payot [des anticipations dans l'article « Histoire et structure », Les Temps modernes 1963, 195 : 281-306].

$1964 c$ « Compte rendu de mission chez les Indiens ayorés du Paraguay et de Bolivie », L'Homme IV (2), mai-août : 126-129.

1965 « Le chamanisme ayoréo », L'Homme V (I), janvier-mars : 7-32 ; (2), avril-juin : 92-122.

1971 L'Invention du monde chez les Indiens pueblos. Paris, Maspero [des extraits d'analyse avaient été anticipés dans l'article « La geste de Kasewat », L'Homme III (2), mai-août 1963 : 22-76, et une partie des conclusions dans l'article « Le mythe, code et message », présentation sous le titre «Lucien Sebag » de Jean-Paul et Marie-Claire Boons, Les Temps modernes 1965, 226 : 1607-1623].

SUSNIK, Branislava

1963 « La lengua de los Ayoweos-Moros. Estructura gramatical et fraseario etnográfico », Boletín de la Sociedad Cientifica del Paraguay y del Museo Etnográfico, Etnolingüistica 8.

\section{NOTES}

1. Jean-Pierre Darmon (1966) a écrit une page très touchante sur les études et la passion politique de Sebag, à Tunis, où il est né et où sa famille vivait.

2. Entre-temps Métraux avait disparu, lui aussi de manière tragique, le 12 avril 1963, pendant que Sebag se trouvait sur le terrain. Sebag avait quitté les Aché et cherché un nouveau terrain, imprévu au départ, probablement suite à des incompréhensions (ou à des approches selon des rythmes de travail différents) avec Clastres. Hélène Clastres s'était associée à la mission chez les Guayaki après avoir rejoint son mari au mois de juillet à São Paulo où il participait avec Sebag à la 
«VIe Réunion brésilienne d'anthropologie» organisée par l'Associação brasileira de antropologia. En septembre ils étaient allés tous les trois dans le Chaco avec la jeep d'un médecin, Carlton Gadjusek (prix Nobel en 1976), qui faisait des prises de sang aux Indiens. Ils visitèrent plusieurs groupes indigènes, y compris des Ayoré qui venaient de se sédentariser dans le Chaco central, mais Sebag continua tout seul vers l'est à Maria Auxiliadora, le long du fleuve Paraguay, où d'autres groupes d'Ayoré avaient été placés par les salésiens dans un domaine acheté par l'Église.

3. Il s'agit de la relation à la réunion de São Paulo (voir note 2). Selon la communication personnelle d'Hélène Clastres, c'est Sebag qui avait travaillé sur ce sujet et présenté la relation, mais il avait voulu que Pierre Clastres la signe lui aussi.

4. La mission salésienne de Maria Auxiliadora devait être abandonnée quelques années plus tard, après des inondations qui détruisirent entre autres les archives salésiennes témoignant de la dynamique des premiers contacts et des décès causés par les maladies que les Blancs avaient importées. Elle céda la place à deux autres villages ayorés , Cucaani et Isla Alta, auxquels un troisième s'est ajouté plus récemment, Guidai Icciai. Dans le Chaco central, on compte actuellement cinq villages : Campo Loro, Ebetogué, Jesudi, Aocojnandi, Tunucojnai, qui sont sous l'autorité des missionnaires protestants. D'autres groupes vivent dans les faubourgs des petites villes mennonites du Chaco. La population ayoré est actuellement d'environ deux mille personnes au Paraguay et de deux mille cinq cents en Bolivie.

5. Sebag entretiendra une correspondance avec cette missionnaire et son mari, Enrique, comme en témoignent les lettres qu'il a reçues dont la dernière est datée du 21 octobre 1964.

6. Quelques éléments nous permettent de le croire, y compris des interviews que nous avons réalisées avec des Ayoré de Bolivie très âgés, rencontrés en juillet 2000 à Campo Loro dans le Chaco paraguayen. Dans une réflexion très intéressante sur l'interprétation de la vibrante -rdans la langue des Zamuco (groupe dans lequel on inscrit les Chamacoco et les Ayoré), Susnik (1963) soutient une opinion différente. Alors qu'au Paraguay « los Ayoweos tratan [...] eludir la vibrante, distinguiéndose así de los otros pueblos imparentados; en la lengua de los Ayorés [en français dans le texte !] de Bolivia abunda la "r" » («les Ayoré essaient [...] d'éluder la vibrante, en se distinguant de cette manière des autres peuples apparentés; dans la langue des Ayoré de Bolivie, le "r" abonde ").

7. Signalons que les Ayoré vendent leurs plumes tressées aussi aux Indiens maha, qui vivent désormais tous à Asunción et qui les utilisent comme élément pour fabriquer d'autres couvrechefs destinés aux touristes.

8. Ce travail a été commencé par Carmen Bernand-Muñoz (1977), à qui Lévi-Strauss confia les cahiers de Sebag, dans son excellente thèse sur Les Ayoré du Chaco septentrional. Étude critique à partir des notes de Lucien Sebag, où la comparaison et la recherche d'analogies et d'inversions structurales concernent surtout les Chamacoco.

9. Une représentation très claire de la «Situation géographique, historique et linguistique des Pueblos » a été écrite par Jacqueline Bolens, comme introduction à L'Invention du monde.

10. Fx (a) : Fy (b) 圈 Fx (b) : Fa - 1 (y) est énoncée pour la première fois dans l'article « La structure des mythes" (Lévi-Strauss 1958). Nous nous limitons ici à rapporter les propriétés fondamentales que Lévi-Strauss lui-même attribue à sa formule : «Deux termes a et $b$ étant donnés simultanément ainsi que deux fonctions, $\mathrm{x}$ et $\mathrm{y}$, de ces termes, on pose qu'une relation d'équivalence existe entre deux situations, définies respectivement par une inversion des termes et des relations, sous deux conditions : $1^{\circ}$ qu'un des termes soit remplacé par son contraire (dans l'expression ci-dessus a et a-1); $2^{\circ}$ qu'une inversion corrélative se produise entre la valeur de fonction et la valeur de terme de deux éléments (ci-dessus : y et a).» (1958: 253) Dans la préface (malheureusement peu citée) à Paroles données, Lévi-Strauss répond ainsi aux critiques de ne pas avoir expliqué ni employé ce qu'en 1955 il avait appelé la "relation canonique [...] à quoi tout mythe est réductible », comme si, implicitement, il donnait raison à ceux qui pensaient qu'elle 
était dénuée de sens: "Or, en dépit de son aspect vaguement algébrique, ma formule ne constituait pas un algorithme permettant d'effectuer des calculs. Je la proposais comme une image ou un dessin: représentation graphique qui, dans ma pensée, pouvait faciliter l'appréhension intuitive d'une chaîne de rapports. Cet effet obtenu, il n'était pas nécessaire de reproduire à tout bout de champ la même figure, pas plus que, dans un livre, on ne reproduit l'illustration d'une scène ou d'un objet chaque fois qu'il arrive à l'auteur d'en parler. D'autant que la relation déséquilibrée, que j'entendais mettre en évidence comme une propriété inhérente aux transformations mythiques, était surabondamment illustrée par mes analyses portant sur des centaines de mythes particuliers." (1984: 13) Ces précisions sont supportées par une démonstration en clair que son cours sur le cannibalisme de 1974-1975 au Collège de France lui permettait. D'autres démonstrations allaient suivre dans La Potière jalouse (1985) et dans Histoire de Lynx (1991).

11. Il est certainement paradoxal que la logique de la prédation (qui n'est d'ailleurs pas une modalité exclusive en Amérique du Sud) soit parfois opposée à celle de l'échange pour soutenir une négation de l'autre que l'unité culturelle du continent américain semble précisément démentir. La question est là : une prédation sans échange serait-elle compatible avec cette unité ? N'existe-t-il pas assez de preuves ethnographiques de formes d'échanges se réalisant par le cannibalisme ou la guerre?

12. La non-utilisation de la formule canonique - ou de la logique qui la sous-tend - dans d'autres aires culturelles aussi vastes que le continent américain n'est pas une preuve d'un manque de validité ni de sa seule validité à l'échelle de ce dernier. Cette limitation régionale de la formule canonique est d'autant plus singulière qu'elle est proposée par ceux qui la rapprochent davantage de la mathématique ou de l'algèbre. Au contraire, l'extension des ressources de la formule à d'autres domaines que le mythe (le rituel ou l'art ethnique par exemple) apparaît très productive. D'ailleurs, c'est Lévi-Strauss lui-même qui l'avait utilisée, pour répondre à ses critiques, dans sa démonstration sur le rituel naven des Iatmul de Nouvelle-Guinée (cf. note 10).

13. Une autre lecture structurale des mythes bien connue par Sebag est celle de «La geste d'Asdiwal » (Lévi-Strauss 1958-1959).

14. Il n'empêche que Sebag propose aussi des similitudes très précises, par exemple entre certains aspects du mythe acoma et le lien entre tabac et animalité dans un mythe d'origine qu'il avait lui-même recueilli chez les Ayoré.

15. Il est intéressant de constater que, par rapport à la linguistique, non seulement Lévi-Strauss suit la même démarche dans les deux domaines de la parenté et du mythe, mais encore qu'à l'atome de parenté ont été adressées les mêmes critiques qu'à la formule canonique. L'utilité de considérer ensemble ces deux aspects de la « révolution copernicienne » que représentent en anthropologie le concept d'atome de parenté et la formule canonique n'a pas encore été explorée à fond.

16. L'article de Benveniste, «Sémiologie de la langue », publié en 1969 dans la revue Semiotica (1: $1-12 ; 2: 127-135)$, reconnaît une des conditions de la signifiance dans le fait que chaque signe soit «pris et compris » dans un système de signes, qu'il y a donc quelque part une différence entre le signe et le signifié. Et cela à l'encontre de Peirce, qui pose le signe comme étant à la base de l'univers entier, de l'homme, de sa pensée, de ses émotions. La préoccupation que la notion de signe puisse disparaître dans cette multiplication à l'infini avait suggéré à Benveniste cet heureux jeu de mots adressé à Peirce : « Mais finalement ces signes, étant tous signes les uns des autres, de quoi pourront-ils être signes qui NE SOIT PAS signe?»

17. Sur cette possibilité d'une analyse panchronique (capable donc de tenir compte des deux axes de la succession et de la simultanéité), Saussure lui-même avait donné quelques indications importantes dans son Cours de linguistique générale.

18. Dans l'intention de Sebag, il semble ne pas s'agir d'une utilisation faible de la formule canonique de Lévi-Strauss, d'une empreinte de vocabulaire, pourrait-on dire. Dans la note 
référant à la formule canonique, il cite les « Éléments de sémiologie » de Roland Barthes (publiés en 1964 dans le $n^{\circ} 4$ de Communications) - où il découvre sans doute l'articulation proposée par Hjelmslev dans sa glossématique (mais en n'utilisant pas la réarticulation des deux plans de l'expression et du contenu en forme et substance) - et l'article de Lévi-Strauss « La structure des mythes» (1958).

19. Le renversement symétrique des termes pour l'expression et le contenu, que Sebag semble concevoir comme un couple de termes contrastants, ouvre vers une logique qui est plus proche du groupe de Klein $(\mathrm{x}-\mathrm{x}, \mathrm{I} / \mathrm{x},-\mathrm{I} / \mathrm{x})$ que de la logique dissymétrique et dynamique de la formule canonique esquissée par Lévi-Strauss. Ces équivalences apparaissent de manière encore plus claire dans le tapuscrit de Sebag car sa formule canonique, qui a été écrite d'abord de la manière

suivante : $\mathrm{E}_{1}$ (e R c) R C est rayée au crayon dans le tapuscrit et remplacée par :

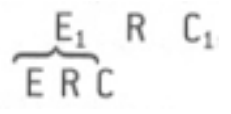
ce qui revient en réalité au même, alors que c'est moins évident dans la version définitive. Dans les Mythologiques, Lévi-Strauss a souvent recours à des groupes (ou à des structures) quadripartites dont les termes sont homogènes et dans un rapport d'exclusion entre eux, mais il analyse également des structures à quatre termes dont la complexité oblige, pour passer d'un terme à l'autre, à une chaine d'opérations plus sophistiquées que celles permises par le groupe de Klein.

20. Il est tout de même significatif que dans ses cahiers de terrain on lise, dans une liste de travaux que Sebag prévoyait de mettre en route à son retour, "Psychanalyse et ethnographie. Nouveaux champs d'analyse mythique ». Les autres travaux projetés, annotés de la date de juin 1964, sont: "Mythologie keresan», «Cannibalisme guayaki», "Rêves de Baipurangi », « Marxisme et ethnographie », " Thèse sur les Moros » (mais aussi « Les thèses »), "L'église de demain».

21. Bien que, dans certains cas, les matériaux eux-mêmes semblent autoriser cette démarche. Mais il s'agit, alors, le plus souvent de mythes aussi bien que de rites et même de formes concrètes d'organisation sociale qui manipulent surtout les relations de parenté en représentant à rebours le passage de la nature à la culture.

22. Dans L'Invention du monde, étrangement, il n'y a aucune référence au double traumatisme que Freud juge nécessaire pour que la névrose s'installe et que Lévi-Strauss dans « La structure des mythes" avait rapproché si étroitement de la formule canonique pour justifier la "double torsion » qui lui est inhérente. De même, on ne trouve qu'une seule référence à Lacan, lorsque Sebag déclare lui avoir emprunté le symbole de la métonymie tiré des formules rendant compte de la « dialectique du désir ». En ce qui concerne les extraits des " conclusions méthodologiques » de L'Invention du monde publiés par Les Temps modernes (le titre "Le mythe : code et message", bien que sebagien, a été donné par la rédaction), il s'agit de la partie finale, malheureusement coupée de toute référence à la formule canonique. Faute d'indications des rédacteurs de la note introductive, nous pensons que cette partie du texte de Sebag n'a été retenue qu'après sa mort.

\section{RÉSUMÉS}

Limité à ce tournant encore peu exploré des études ethnologiques qui chevauche les années 1960, cet article explore quelques aspects de la transition de Lucien Sebag de sa formation philosophique à la théorie ethnologique, transition dont les piliers les plus importants sont 
l'apprentissage auprès de Claude Lévi-Strauss et ses enquêtes de terrain chez les Aché-Guayaki de la zone orientale du Paraguay et chez les Ayoré-Moros du Chaco paraguayen et bolivien. L'article met particulièrement en lumière la contribution de Sebag à l'analyse structurale des mythes et, par le dépassement de l'approche marxiste et de la démarche psychanalytique, les nouvelles perspectives qu'en accord avec la linguistique structurale il avait commencé à ouvrir en anthropologie.

This article describes the largely ignored ethnological studies carried out around the 1960s. It covers some of the aspects of Lucien Sebag's conversion from philosopher to ethnological theorist, a transition which stemmed in particular from his work with Claude Lévi-Strauss and field studies of the Aché-Guayaki in eastern Paraguay and of the Ayoré-Moros in Paraguayan and Bolivian Chaco. The article highlights Sebag's contribution to the structural analysis of myth and, going beyond the Marxist and psychoanalytical approach, the new perspectives based on structural linguistics that he started to open in anthropology.

\section{INDEX}

Keywords : Aché-Guayaki, Ayoré, canonic formula, myth, structural analysis

Mots-clés : Aché-Guayaki, analyse structurale, Ayoré, formule canonique, mythe

\section{AUTEUR}

\section{SALVATORE D'ONOFRIO}

Université de Palerme / LAS, s.donofrio@libero.it 